\title{
Flicker induces depth: Spatial and temporal factors in the perceptual segregation of flickering and nonflickering regions in depth
}

\author{
EVA WONG and NAOMI WEISSTEIN \\ State University of New York, Buffalo, New York
}

\begin{abstract}
If some regions of a random-dot field are flickered, then the nonflickering areas appear to stand out in depth in front of the flickering regions. This perception of depth is optimal within a limited range of temporal frequencies. The average temporal luminance of the flickering and nonflickering regions was kept equal, so the depth segregation is not due to a luminance difference. In fact, depth is seen even when the average temporal luminance of the flickering regions is twice that of the steadily presented regions. The magnitude of perceived depth is affected by the percentage of luminance modulation: depth is maximal at $100 \%$ modulation and diminishes as the percent modulation decreases. We charted the tuning function using alternating flickering and nonflickering random-dot bars and found it to be similar to those of visual channels most sensitive to high temporal frequency.
\end{abstract}

If areas of a filled visual field are flickered, the flickering regions appear to lie in depth well behind the nonflickering regions (Wong \& Weisstein, 1983a). When parts of a random-dot field were flickered so that the display consisted of alternating flickering and nonflickering "bars" composed of dots, the nonflickering "bars" were seen to stand out in front of the flickering "bars."

The nonflickering "bars" appear to form coherent rectangular shapes located in front of a background of flickering dots. The impression is not different from that of a row of figures contrasted against a flickering backdrop. The perceived depth effect reminds one of random-dot stereograms in which an area of dots may appear to float out in space in front of a background. Figure 1 is a representation of what the percept looks like to an observer. The flickering areas appear to stay at a constant depth behind the nonflickering areas rather than receding and approaching with the on-and-off cycle of the flicker. In other words, once the area is flickered at the appropriate temporal frequency, the flickering area remains steadily at a constant depth behind the nonflickering areas.

The depth segregation produced by flicker-flickerinduced depth -is not due to a luminance difference between the flickering and nonflickering areas, since the average temporal luminance of all the regions was kept equal. It is also not dependent on the textural elements making up the visual field, since we obtained the effect with dots, horizontal lines, and

This research was supported by NEI Grant R01 EY03432 to Naomi Weisstein. The authors' mailing address is: Department of Psychology, SUNY, 4230 Ridge Lea Road, Buffalo, NY 14226.

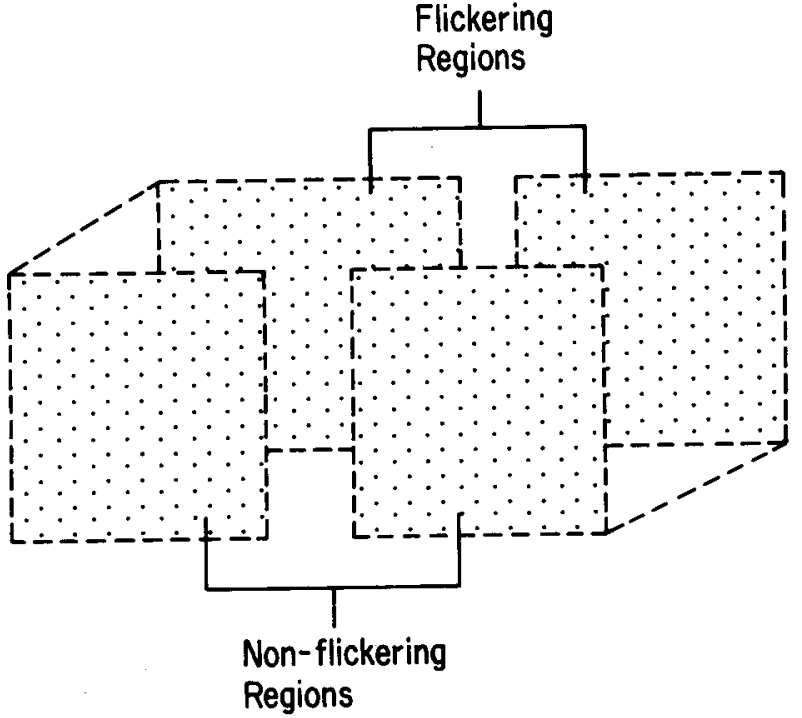

Figure 1. Perceptual impression of nonflickering areas lying in front of a flickering background. (Dashed lines are used to mark regions for purposes of illustration. They did not exist in the display used in the experiment.)

vertical lines; nor does it depend on a specific configuration of the flickering and nonflickering regions, since we obtained this effect with "bars" made up of different textures and with concentric squares. The effect is not dependent on the density of dots filling the flickering and nonflickering regions, provided there is an adequate number of dots to define a region. Moreover, we found that a temporal frequency of about $6 \mathrm{~Hz}$ produced the greatest depth separation between the flickering and nonflickering areas, suggesting that visual channels responding pri- 
marily to high temporal frequencies might be involved in segregating perceptual regions in depth.

At first glance, this flicker-induced depth may recall the Gestalt organization principle "grouping by common fate" (Johansson, 1950; Wertheimer, 1923). (Johansson has demonstrated that dots moving in the same direction are grouped together.) However, a closer examination reveals that our phenomenon is not just an instance of grouping by flicker, analogous to "grouping by common fate." First, in our case flicker produces depth as well as segregation of the perceptual field into defined regions, whereas "grouping by common fate," typically defined, does not involve depth. Second, our phenomenon is optimal around a temporal frequency of $6 \mathrm{~Hz}$, showing an effect of tuning, whereas "grouping by common fate," for example grouping by motion, is not dependent on the velocity of the moving elements (Johansson, 1950). Finally, we have some preliminary findings showing that when a region is flickered at very low temporal frequencies (below $2 \mathrm{~Hz}$ ), grouping of the flickering and nonflickering elements can still be experienced, but the depth separation between those regions has disappeared. Thus, although "grouping by flicker" might be the basis for segregating the elements into defined regions, certain rates of temporal modulation are necessary for perceiving the flickering and nonflickering areas as segregated in depth.

The three experiments we report in this paper are further investigations of the depth segregation produced by flicker. The first experiment examined the spatiotemporal tuning of the flicker-induced depth using bars composed of alternating flickering and nonflickering random dots. The second experiment examined the effect of the amplitude of temporal modulation on the amount of perceived depth separation between the flickering and nonflickering areas. In the third experiment, we looked at how luminance differences between the flickering and nonflickering regions might affect the amount of depth perceived.

\section{EXPERIMENT 1}

This experiment was designed to investigate the spatiotemporal tuning of depth segregation produced by flicker.

\section{Method}

Subjects. Seven naive observers from an undergraduate subject pool participated in this experiment. All had 20/20 vision.

Stimuli and Apparatus. The display consisted of a field of random dots covering an area of $5.4 \times 2.7 \mathrm{deg}$ (see Figure 2). Regions of this random-dot field were flickered to form a "grating" composed of alternating flickering and nonflickering "bars." All "bars"' were equal in width. There were 9 bar widths $(2.7,1.35$, $1.0, .68, .54, .34, .27, .21, .14$ degrees) and 12 temporal square-

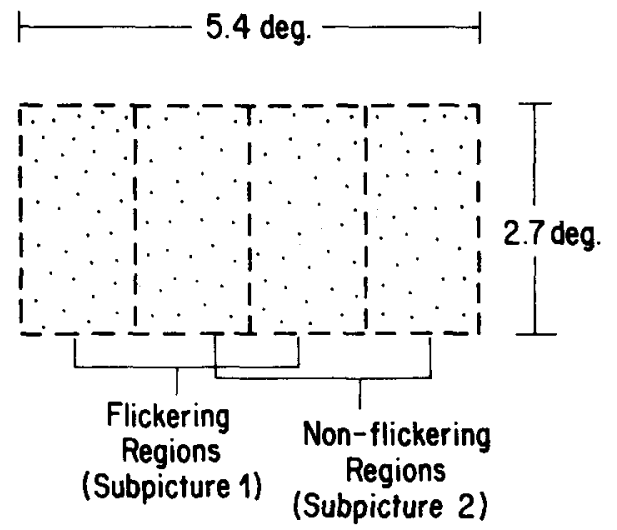

Figure 2. Illustration of the display used in the experiments. This is an example of flickering and nonflickering bars composed of random dots with a bar width of $.68 \mathrm{deg}$.

wave frequencies $(1,1.4,2,2.8,3.6,5,5.5,6.3,7.1,8.3,10$, $12.5 \mathrm{~Hz}$ ).

A PDP-11 computer with a GT-40 graphics processor generated the stimuli and controlled the experiment. The entire randomdot field was composed of two subpictures-the flickering regions and the nonflickering regions. The computer first selected the "bar" width from a list containing the randomized order of the bar widths to be used in the experiment, calculated the screen coordinates bordering each "bar," and tagged each "bar" as either "flickering" or "nonflickering." The "flickering" and "nonflickering" regions were then filled with random dots to form two respective subpictures, one of the subpictures to be made to flicker and the other to remain steady on the screen (see Figure 2). The intensity of the dots in the flickering areas was set so that during flicker, the average temporal luminance of the flickering and nonflickering regions was kept equal. (The GT-40 display processor has eight intensity levels, and each subpicture can be generated with a specified intensity level.) The mean luminance $\left(L_{\text {mean }}\right)$ is defined in terms of $L_{\max }+L_{\min } / 2$, where $L_{\max }$ and $L_{\min }$ are, respectively, the maximum and minimum luminances in the temporal cycle. The temporal modulation amplitude was set at $100 \%$ in this experiment, and square-wave flicker was used throughout. Flickering was achieved by turning on and off a subpicture. The on/off cycle defined the temporal frequency.

Procedure. Subjects viewed the display from a distance of $1 \mathrm{~m}$. Viewing was monocular; the left eye was occluded. At the beginning of each trial, the subject fixated a fixation mark (a cross) in the center of the screen. When ready, the subject pressed a key to initiate the trial. The computer selected a certain bar width randomly, generated the display, randomly picked a flicker rate out of the 12 frequencies, and presented the flickering stimulus for $10 \mathrm{sec}$. The subjects were all naive to the purpose of the experiment. They were told only that they would view a display consisting of flickering and nonflickering dots and that they were to report their subjective impressions of the display. The subjects first reported their subjective impressions about the display presented. If their responses indicated that they perceived the flickering and nonflickering areas as segregated into distinct perceptual regions as well as separate in depth, then they were asked to make further quantitative judgments. A horizontal line appeared on the screen, and the subject instructed the experimenter to adjust its length so that it matched the amount of perceived depth separation between the flickering and nonflickering areas. The direction of depth was signed $(+)$ if the nonflickering regions were perceived to be in front of the flickering regions. $A(-)$ was signed if the reverse occurred. Each bar width and temporal frequency combination was presented five times. Each data point on the tuning function 
was thus based on the mean of seven observers' each making judgments from five exposures.

\section{Results}

First, no observer perceived the flickering areas in front of the nonflickering areas for any spatial and temporal frequency combinations (binomial, $\mathrm{p}<$ .001 ). In other words, if any depth separation was seen between the flickering and nonflickering areas, the steady regions were always seen in front. Second, the observers perceived the flickering and nonflickering dots segregated into definite perceptual regions regardless of whether depth separation was seen (binomial, $\mathrm{p}<.001$ ). Third, the observers' judgments were very consistent within stimulus conditions. Within observers, with each observer making five judgments from five exposures per stimulus condition, the standard deviation did not exceed 0.5 .
Across subjects, the standard deviation of the depth judgments did not exceed 0.9. Lastly, we were able to obtain a definite tuning function for the depth segregation produced by flicker.

Figure 3 shows the tuning function displayed in a two-dimensional plot. Each point defined by $x-y$ coordinates represents a locus in the tuning function. The isodepth contours joining the coordinate points connect spatial (bar width) and temporal loci giving a similar level of depth separation to be perceived between the flickering and nonflickering areas. Each contour (or level of perceived depth) was arrived at in the following way. First, the response measures (adjustment of the length of a horizontal line) from the seven subjects for each spatial and temporal frequency combination were averaged. The computer found the six largest gaps among the judgment values. Gap sizes two standard deviations below the

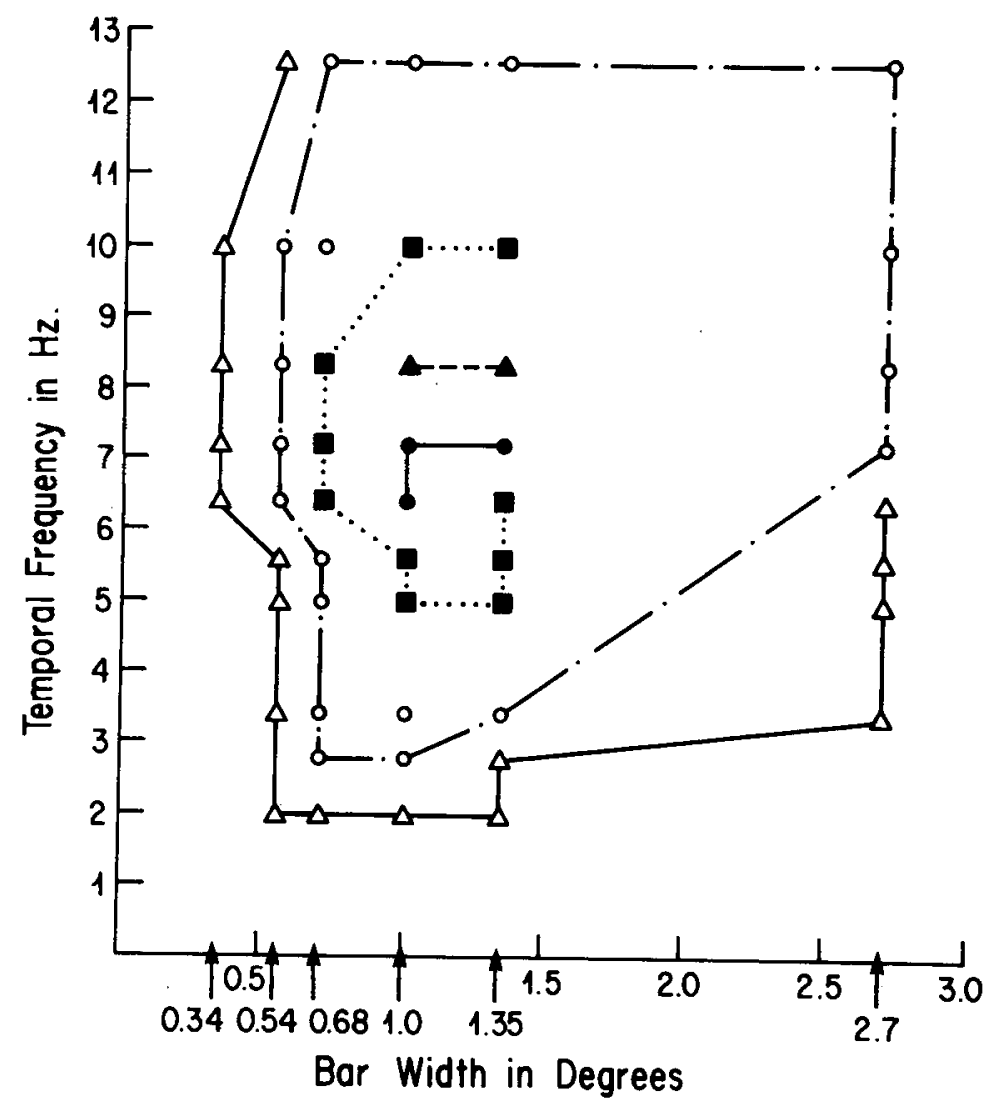

Depth Magnitude in $\mathrm{cm}$.
$\Delta 0.05-0.14$
- $0.35-0.44$
$0.80-0.90$
$\Delta 1.30-1.40$
- $2.00-2.40$

Figure 3. Tuning function for depth segregation Induced by ticker. Contours connect bar width and temporal frequency loci where a similar amount of depth is percelved. 
mean were dropped; the remaining gaps were then used to define clusters of depth values. Five clusters emerged from the data collected. (See Scher, Shneier, \& Rosenfeld, 1982, for a discussion and evaluation of this and other methods of cluster extraction.) Within each of the five contours defined by clustering, no subject's judgment fell out of that contour.

From Figure 3, it is clear that large bar widths and high temporal frequencies produced the maximum depth separation between the flickering and nonflickering regions. At temporal frequencies of 6.3 and $7.1 \mathrm{~Hz}$ and bar widths of 1.35 and $.68 \mathrm{deg}$, the depth effect is greatest. The upper spatial limit (in terms of bar width) is bounded by $2.7 \mathrm{deg}$, and the depth effect disappears below a temporal frequency of $2 \mathrm{~Hz}$. Depth is perceived up to the highest flicker rate used in this experiment $(12.5 \mathrm{~Hz})$, although it is substantially diminished. At the lower end of smaller bar widths, depth segregation occurs down to $.34 \mathrm{deg}$.

\section{EXPERIMENT 2}

This experiment examined the effect of amplitude of temporal modulation on depth segregation between flickering and nonflickering regions. The spatial (bar width) and temporal frequency range that had given the optimal depth response in Experiment 1 was used.

\section{Method}

Subjects. Seven new, naive observers from the undergraduate subject-pool participated in this experiment. All had 20/20 vision.

Stimuli and Apparatus. The display was the same as that of Experiment 1. Four bar widths of alternating flickering and nonflickering dots $(2.7,1.35,1.0$, and $.68 \mathrm{deg})$ and five temporal frequencies of square-wave flicker $(5,5.5,6.3,7.1$, and $8.3 \mathrm{~Hz})$ were used. These were the spatial and temporal parameters that gave sizable amounts of depth separation between the flickering and nonflickering regions. The flickering regions were modulated at amplitudes of $25 \%, 50 \%, 75 \%$, and $100 \%$. Amplitude of modulation is defined as $\mathrm{L}_{\max }-\mathrm{L}_{\min } / \mathrm{L}_{\max }+\mathrm{L}_{\min }$. At $100 \%$ modulation, the stimulus is identical to that in Experiment 1. The average temporal luminance across all spatial regions and at all the modulation amplitudes was kept equal.

The display was generated using the methods described in Experiment 1 , except that an additional procedure was needed to simulate the different percentages of temporal modulation. This was achieved in the following way. The nonflickering regions were generated as one subpicture (as in Experiment 1). The flickering regions now consisted of two identical subpictures superimposed on each other. Their intensities were set so that when both of them were turned on, the total luminance equaled the maximum luminance $\left(L_{m a x}\right)$ in the cycle. When one of them was turned on, intensity equaled that of the minimum in the cycle $\left(L_{\text {min }}\right)$. Thus, by changing the intensities of the two subpictures making up the flickering regions, modulation amplitudes of $25 \%$, $50 \%$, and $75 \%$ can be simulated. At $100 \%$ modulation, one subpicture was set at zero intensity.

Procedure. Viewing conditions were similar to those in Experiment 1 . Combinations of bar width, temporal frequency, and modulation amplitude were presented in randomized order. Data were collected as in Experiment 1 . The instructions to the subjects were the same as those in Experiment 1.

\section{Results}

First, no observer perceived the flickering regions in front of the nonflickering regions for any modulation amplitudes across all the bar width and temporal frequencies used in this experiment (binomial, $p<$ $.001)$. In other words, if any depth separation was seen between the flickering and nonflickering areas, the steady regions were always seen in front. Second, all subjects perceived the flickering and nonflickering dots as being segregated into definite perceptual regions regardless of whether depth separation was perceived between them (binomial, $\mathrm{p}<.001$ ). Third, within-subjects and between-subjects judgments were very consistent, with the standard deviation less than 0.8 . Across subjects, the standard deviation of the depth judgments did not exceed 0.9. Lastly, we found that amplitude of temporal modulation affects the magnitude of perceived depth separation between the flickering and nonflickering areas. In Figure 4, panels $A, B$, and $C$ show the spatiotemporal tuning functions of the depth effect for $100 \%, 75 \%$, and $50 \%$ modulation, respectively. At $25 \%$ modulation, no depth was perceived, although the flickering and nonflickering dots were segregated into distinct perceptual regions.

As in Experiment 1, the isodepth contours here were defined by clusters of values from the response measures. Five clusters were extracted from the judgment values (from four bar widths, five temporal frequencies, four amplitudes of temporal modulation), each of which was based on the average of seven observers' making five adjustments. Within each of the five contours, no subject's depth judgment fell out of that contour.

From Figure 4, it is clear that perceived depth separation between flickering and nonflickering regions was maximal at $100 \%$ modulation. Data from this condition follow trends similar to those of Experiment 1 at the same temporal frequencies and bar widths, thus replicating the findings of the previous study with different subjects. At $75 \%$ modulation, depth segregation diminished. At the bar widths and temporal frequencies at which depth was maximum at $100 \%$ modulation (1.35 and $.68 \mathrm{deg} ; 6.3,7.1$, and $8.3 \mathrm{~Hz}$ ), the amount of perceived depth had dropped by an average of at least $1 \mathrm{~cm}$. At spatiotemporal regions where depth was marginal at $100 \%$ modulation $(.34 \mathrm{deg}$, and below $6.3 \mathrm{~Hz}$ ), no depth segregation was perceived at $75 \%$. In terms of contours of depth judgments, the level of depth values had dropped three steps, from the highest level down to the second lowest level. At $50 \%$ modulation, the amount of depth separation decreased by an average of about $2 \mathrm{~cm}$ at bar widths and temporal frequencies at which depth was maximum at $100 \%$ modulation. In regions in which depth was minimal at $75 \%$ modulation, depth segregation disappeared at $50 \%$ modu- 


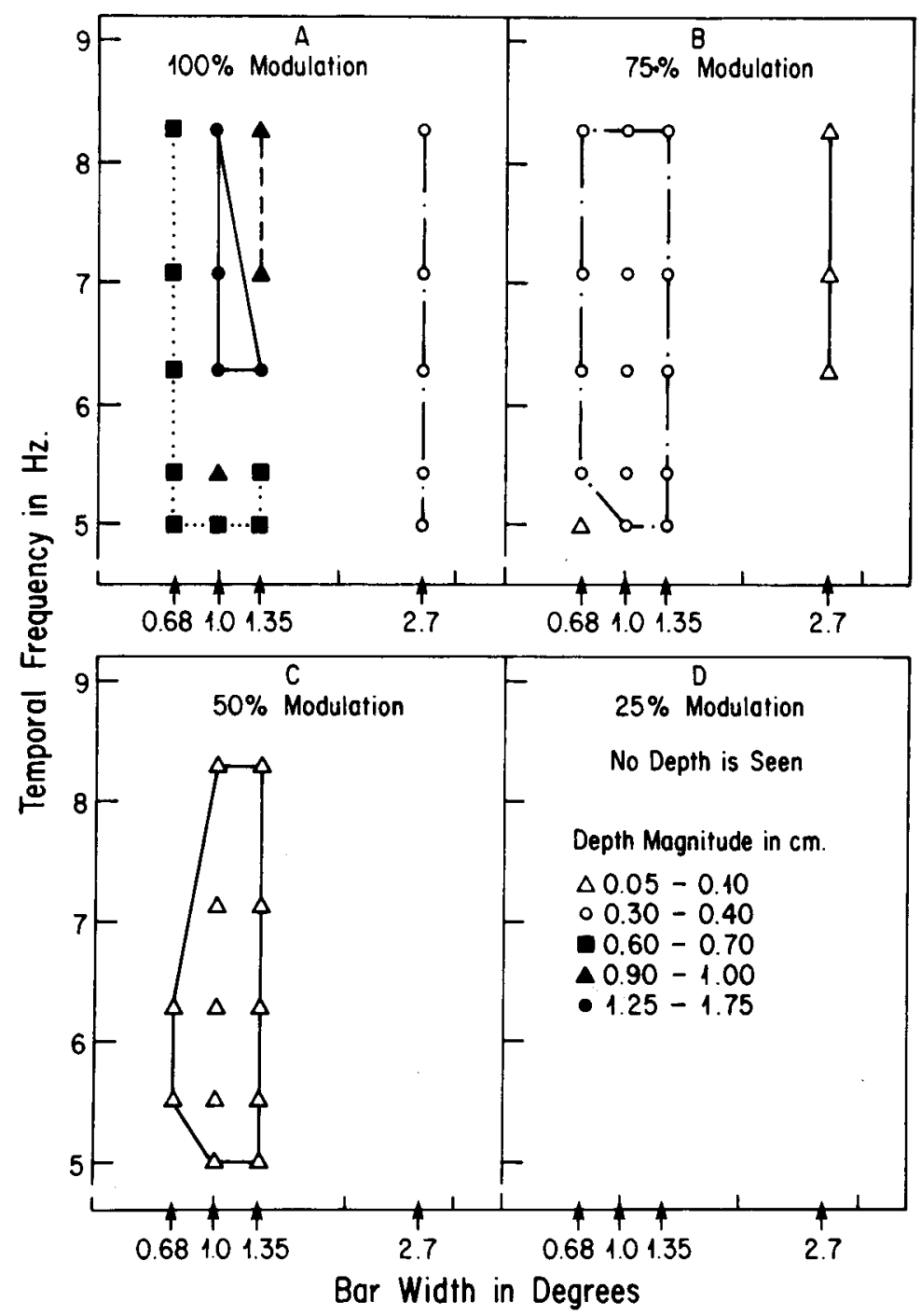

Figure 4. Comparison of tuning functions of the depth effect induced by flicker at temporal modulation amplitudes of $100 \%, 75 \%, 50 \%$, and $25 \%$.

lation. At $25 \%$ modulation, no depth was seen at all in the stimulus, although segregation of the random dots into flickering and nonflickering regions was perceived. In fact, the striking feature of Figure 4 emerges in the comparison of the three panels (A, B, C): disappearance of the isodepth contours representing high depth values as depth of modulation decreases, and encroachment of contours representing low depth values into those areas formerly occupied by contours of high depth values.

A three-way ANOVA (repeated measures) showed all three main effects to be significant [bar-width factor, $F(3,18)=15.81, p<.001 ;$ temporal frequency factor, $F(4,24)=13.78, p<.001$; amplitude of modulation, $F(3,18)=27.55, p<.001]$. All the two-way interactions were significant [bar width $\times$ temporal frequency, $\mathrm{F}(12,72)=2.54, \mathrm{p}<.01$; bar width $\times$ modulation amplitude, $F(9,54)=2.07, p<.05$; temporal frequency $\times$ modulation amplitude,
$\mathrm{F}(12,72)=1.96, \mathrm{p}<.05]$. The three-way interaction tended toward significance at the .05 level, but did not reach the statistical criterion $[F(36,216)=0.94]$.

\section{EXPERIMENT 3}

In this experiment, we examined the effect of luminance differences on the amount of depth perceived between flickering and nonflickering regions. The luminance of the flickering areas was varied while the luminance of the nonflickering areas was kept constant.

\section{Method}

Subjects. Seven new, naive observers from the undergraduate subject pool participated. All had 20/20 vision.

Stimull and Apparatus. The display was the same as that used in Experiments 1 and 2. The four bar widths and five flicker rates were those used in Experiment 1. The display was generated using the same procedures described for Experiment 1, except that the 
intensity parameter for the flickering regions was varied to give the following luminance differences between the flickering and nonflickering regions (defined by the ratio flickering/nonflickering): $.75,1,1.25,1.5,1.75$, and 2 . When the ratio was 1 , the average temporal luminance between the flickering and nonflickering areas was matched; when the ratio was 1.5 , the average temporal luminance of the flickering region was one and a half times that of the average temporal luminance of the nonflickering regions. Square-wave flicker was used throughout, and the amplitude of modulation was always set at $100 \%$. As in the other experiments, the display was exposed for $10 \mathrm{sec}$ in each trial.

Procedure. Viewing conditions were similar to those of Experiments 1 and 2. Combinations of bar widths and temporal frequency, and luminance ratios between flickering and nonflickering regions, were presented in randomized order. Data were collected using the method described in the other experiments except that, in addition to making region-segregation and depth judgments, the subject also judged whether the flickering areas appeared dimmer, brighter, or equally as bright as the nonflickering regions. The instructions to the subjects were the same as those given in Experiments 1 and 2.

\section{Results}

First, no subject perceived the flickering regions in front of the nonflickering regions for any of the luminance ratios across the bar widths and temporal frequencies used in this study (binomial, $p<.001$ ). In other words, if any depth separation was seen between the flickering and nonflickering areas, the steady regions were always seen in front. Second, all subjects also perceived flickering and nonflickering dots segregated into distinct perceptual regions regardless of whether depth separation was seen between the two segregated areas (binomial, $\mathrm{p}<.001$ ). Third, judgments within subjects and between subjects for a given stimulus condition were very consistent, with the standard deviations less than 1.0.

The effect of luminance difference between the flickering and nonflickering fields on the amount of perceived depth separation between the regions is shown in Figure 5. Data for each luminance ratio (flickering/nonflickering) are shown in the six panels.

The tuning functions shown in Figure 5 were generated with the procedure described in Experiment 1. Five clusters emerged from the depth judgments made by the subjects. These are represented in the figure as isodepth contours connecting temporal frequencies and bar widths, giving a similar amount of depth segregation between the flickering and non-

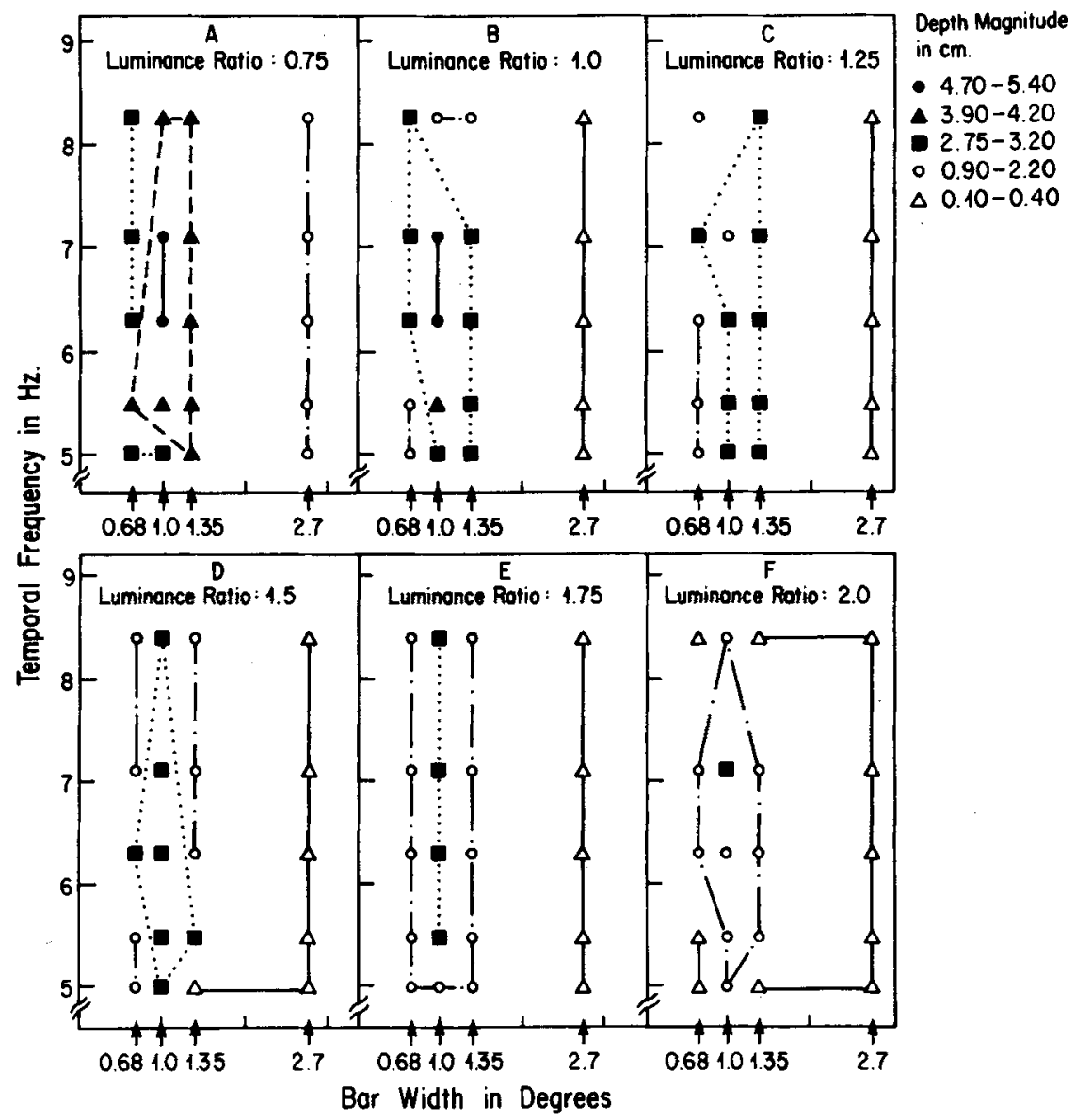

Figure 5. Comparison of tuning functions of the depth effect induced by filcker at six luminance differences between the filckering and nonflickering regions. The ratios are expreased as filckering areas/nonflickering areas. 
flickering regions. Within each contour, no subject's depth judgment fell outside that contour.

When the flickering regions had an average temporal luminance .75 times that of the nonflickering regions' temporal luminance, the depth separation between the two areas was greatest (see panel A). A substantial amount of depth was also seen across all the temporal frequencies and bar widths used. In this condition, six of seven observers perceived the flickering regions to be dimmer than the nonflickering regions. Only one observer judged all the regions to be equally bright. Not much difference existed between the spatiotemporal tuning functions at luminance ratios of 1, 1.25, and 1.5 (panels B, C, and D in Figure 5).

When the ratio was 1 , all the observers perceived all the regions as equally bright at the lower temporal frequencies. Two subjects judged the flickering regions to be brighter than the nonflickering regions at the high temporal frequencies (above $7.1 \mathrm{~Hz}$ ). At luminance ratios 1.25 and 1.5 , all observers reported the flickering regions as brighter than the nonflickering regions, although the latter were perceived to stand out in front. When the average luminance of the flickering regions was 1.75 and 2 times that of the nonflickering regions, the amount of perceived depth separation between these areas diminished. At bar widths and temporal frequencies at which depth was maximum in the original (equal luminance) condition, the magnitude of depth now dropped by an average of $1.5 \mathrm{~cm}$. When the luminance ratio was 1.75 , sizable depth was perceived only at a bar width of $1.0 \mathrm{deg}$ across $5.5,6.3,7.1$, and $8.3 \mathrm{~Hz}$. At the other spatiotemporal regions, perceived depth segregation was weak. At a luminance ratio of 2 , the amount of perceived depth was minimal across all the bar widths and temporal frequencies. Maximum depth perceived here amounted to no more than $1.7 \mathrm{~cm}$; in that same bar width and temporal frequency range for the smaller luminance ratios $(1,1.25,1.5)$, the maximum depth perceived was $3.8 \mathrm{~cm}$.

A three-way ANOVA (repeated measures) showed all main effects to be significant [bar width factor, $F(3,18)=16.81, p<.001$; temporal frequency factor, $\mathrm{F}(4,24)=14.64, \mathrm{p}<.001$; luminance ratio, $\mathrm{F}(5,30)=$ $4.86, \mathrm{p}<.005]$. All the two-way interactions were significant [bar width $\times$ temporal frequency, $F(12,72)=$ $2.94, p<.005$; bar width $\times$ luminance ratio, $F(18,90)$ $=2.88, \mathrm{p}<.005$; temporal frequency $\times$ luminance ratio, $F(30,120)=1.52, p<.01]$. The three-way interaction tended to approach significance but did not reach the statistical criterion $[F(12,270)=0.86]$.

A post hoc comparison between means for a factorial design was performed on the luminance ratio factor. We decided to compare means within category rather than to make comparisons between specific cells, although the interactions in the ANOVA were significant. This is because the issue of interest here was to assess the contribution of particular luminance ratios to the overall effect of luminance dif- ference on the perceived amount of depth separation between the flickering and nonflickering regions. A comparison of specific cells would fail to highlight this point. Means for the luminance ratios .75, 1.75, and 2 were all significantly different from the rest of the ratios. No significant differences were found among the means for the ratios $1,1.25$, or 1.5.

We also wanted to know whether perceived brightness of the flickering areas was related to the magnitude of perceived depth separation between the flickering and nonflickering regions, especially when the luminance ratio was held constant. We therefore correlated the depth judgment (indexed by adjustment of a line's length) and the brightness comparison made between the flickering and nonflickering areas (indexed by three categorical responses of brighter, dimmer, and same). A multiple-covariate ANCOVA was performed on the data. Magnitude of perceived depth was correlated with the independent variable brightness judgment, when bar width, temporal frequency, and luminance ratio are covariates. The $F$ ratio was found to be significant at the .05 level $[F(2,15)=3.78]$.

It should be noted that, at the luminance ratios of 1.75 and 2, although the magnitude of depth separation between the nonflickering and flickering areas diminished and the former was perceived as bright, no observer saw the flickering regions in front of the nonflickering regions.

\section{GENERAL DISCUSSION}

\section{Flicker and Depth}

Flickering a region of a filled visual field segregates it in depth behind areas that are not flickered. This depth segregation was found to be strongly dependent on temporal frequency and width of the bars in our display, thus establishing it as an effect related to temporal frequency rather than merely to grouping due to flicker. It is known that temporal changes are often associated with depth cues. Clusters of dots moving in the same direction are readily segregated from a field of stationary dots (Julesz, 1971). If dots in both areas move, the faster moving area is more prominently seen as a figure. In addition, if the slower moving dots become hidden when they approach the faster moving cluster, then the powerful depth cue of interposition can make the faster moving cluster appear in depth in front of the slower moving background. Recently, Allman, Campbell, and McGuinness (1979) and Frost, Morgan, and Nakayama (1982) have confirmed this finding. Thus, motion, occlusion, and motion sheering off edges can produce strong effects of area segregation in depth. Another depth phenomenon associated with motion is the segregation of two overlapping fields of dots moving at different velocities (Farber \& McConkie, 1979). The field that was perceived as moving faster was almost invariably seen as lying in front of the 
field that was perceived as moving more slowly. These depth effects, produced by differential velocities, are most probably related to the depth signaled by motion parallax. Fast optical flow is associated with fields nearer the observer, and slow flow is associated with fields located farther away.

Can our flicker-induced depth be subsumed as a phenomenon related to motion-associated depth cues? We think not. In our flicker-induced depth, it is the nonflickering (or static) areas that are seen in front, not the areas that have a high rate of temporal change. Moreover, the amount of depth segregation perceived is tuned to temporal frequency (or flicker rate), where frequencies between 6.3 and $8.3 \mathrm{~Hz}$ give the largest amount of perceived depth segregation. This makes it different from depth signaled by differential velocities of moving fields related to motion parallax. Similarly, our effect cannot be accounted for in terms of occlusion cues, even if the temporal change (flicker) adjacent to a stationary edge is considered as a cue indicating disappearance behind opaque regions. One would expect occlusion to work whether the temporal change at a stationary edge was fast or slow. In fact, for occlusion to function as a reliable cue for depth, it must be independent of the rate of temporal change. If occlusion were tied to temporal tuning, then only objects moving at certain velocities would be seen as disappearing behind other objects. This would defeat the whole purpose of occlusion as a depth cue.

\section{Apparent Brightness and Magnitude of Perceived Depth}

Luminance differences between the flickering and nonflickering regions also determine the amount of depth separation seen between these areas. However, this was true here only when the average luminance of the flickering regions was lower or substantially higher than that of the nonflickering regions. This is not surprising, since regions with a high luminance level were also perceived as subjectively brighter, and it is known that subjective brightness of a stimulus affects its perceived depth (Ittelson, 1960). Dimmer objects are judged as farther away than bright objects. Perceived brightness as a cue to depth may counteract the depth effect produced by flicker when the flickering areas are subjectively brighter than the nonflickering areas, and enhance depth when the reverse is true. It is important to note, though, that subjective brightness cannot be the explanation for the depth segregation perceived, since flickering regions that were judged as brighter were still localized behind the dimmer nonflickering areas.

\section{Figure-Ground and Flicker-Induced Depth}

It is known that figure-ground segregation is accompanied by an impression that figure and ground lie on different depth planes (Coren, 1972; Hochberg, 1972; Kanizsa, 1979; Koffka, 1935). A figure is often seen in front of backgrounds, and regions that are localized nearer the observer are often designated as figures (Julesz, 1971). Our phenomenon of flickerinduced depth is strongly suggestive of figure-ground segregation. In our experiments, observers often described the nonflickering regions as "bars emerging out as shapes in front of a background made of flickering dots." Thus, the nonflickering regions are designated as figures, while the flickering areas are seen as a sheet of dots extending behind, and partially hidden by, the nonflickering bars. That the flicker-induced depth is temporally tuned may suggest the involvement of temporal frequency channels in the segregation of figure and ground. Recently, we have found evidence that flickering a region at the same temporal frequencies at which flicker-induced depth is most prominent also causes a region of an ambiguous picture to be seen as background most of the time (Wong \& Weisstein, 1983b). This mounting evidence makes it reasonable to think that the temporal frequency response, flickerinduced depth, and figure-ground segregation are closely related.

\section{REFERENCES}

Allman, J., Campeell, C. B. G., \& McGuinness, E. (1979). The dorsal third tier area in Galugo senegalensis. Brain Research, 179, 355-361.

Coren, S. (1972). Subjective contours and apparent depth. Psychological Review, 79, 359-367.

FARber, J. M., \& MCConkie, A. B. (1979). Optical motions as information for unsigned depth. Journal of Experimental Psychology: Human Perception and Performance, 5, 494-500.

Frost, B. J., Morgan, B., \& Nakayama, K. (1982). Single tectal cells sensitive to differential motion independent of direction. Supplement of Investigative Ophthalmology and Visual Science, 22, 12.

Hochbe RG, J. (1972). Color and shape. In L. A. Riggs \& J. F. Kling (Eds.), Woodsworth and Scholsberg's Experimental psychology (3rd ed.). New York: Holt, Rinehart \& Winston.

ITtelson, W. H. (1960). Visual space perception. New York: Springer.

Johansson, G. (1950). Configurations in event perception. Uppsala: Almkvist and Wiksell.

Julesz, B. (1971). The foundations of cyclopean perception. Chicago: University of Chicago Press.

Kanizsa, G. (1979). Organization in vision. New York: Praeger.

KoffKA, K. (1935). Principles of Gestalt psychology, New York: Harcourt, Brace.

Scher, A., Schneier, M., \& Rosenfeld, A. (1982). Clustering of collinear line segments. Pattern Recognition, 15, 85-92.

Wertheimer, M. (1923). Untersuchungen zur Lehre von der Gestalt, II. Psychologische Forschung, 4, 301-350.

Wong, E., : Weisstein, N. (1982). A new context-superiority effect: Line segments are more visible against a figure than against a ground. Science, 218, 587-589.

Wona, E., \& WeIsstein, N. (1983a). A new phenomenon in perceptual organization:Flickering fields are localized in a depth plane behind non-flickering fields. Manuscript submitted for publication.

Wona, E., \& Weisstein, N. (1983b). Flickering regions of reversible pictures are seen as backgrounds and non-flickering re. gions are seen as figures. Manuscript in preparation.

(Manuscript received August 26, 1982; revision accepted for publication December 12,1983 ). 